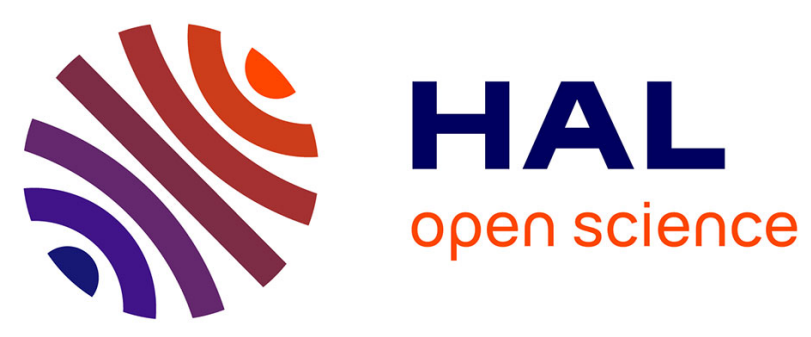

\title{
Pour des droits de la Nature
}

Fabrice Flipo

\section{To cite this version:}

Fabrice Flipo. Pour des droits de la Nature. Mouvements : des idées et des luttes, 2012, 70, pp.122-137. 10.3917/mouv.070.0122 . hal-00958091

\section{HAL Id: hal-00958091 \\ https://hal.science/hal-00958091}

Submitted on 11 Mar 2014

HAL is a multi-disciplinary open access archive for the deposit and dissemination of scientific research documents, whether they are published or not. The documents may come from teaching and research institutions in France or abroad, or from public or private research centers.
L'archive ouverte pluridisciplinaire HAL, est destinée au dépôt et à la diffusion de documents scientifiques de niveau recherche, publiés ou non, émanant des établissements d'enseignement et de recherche français ou étrangers, des laboratoires publics ou privés. 


\section{Pour des droits de la nature}

Par Fabrice Flipo

La revendication de « droits de la nature » émerge dans l'espace international, dans le contexte de Rio+20. Fin 2011 la Bolivie a fait une proposition portant explicitement ce titre, et situant sa contribution dans la droite ligne de textes passés : la Charte mondiale pour la nature (1982), la Déclaration de Rio (1992), la Charte de la Terre (2000) et le Sommet des peuples sur le changement climatique et les droits de la Terre-Mère, à Cochabamba (2010). Le chapitre 7 de la nouvelle constitution équatorienne de 2008 évoque l'existence de « droits de la nature ». La revendication a été reprise par divers mouvements sociaux, non sans quelques remous. Ainsi Pierre Khalfa de Solidaires déclarait-il en août 2010 que les droits de la nature sont « une absurdité » (Libération, 23 août 2010). Qu'en penser ? Les « droits de la nature " sont-ils une fausse piste, menant à une remise en cause de la modernité, en lieu et place de l'émancipation ? Et si la modernité était précisément ce qui fait obstacle à l'universalité, au contraire ?

I. Les droits de la nature, un élément nouveau dans les relations internationales ?

Examinons tout d'abord ce que disent ces textes, à commencer par les propositions boliviennes et équatoriennes pour Rio +20 , qui ont été déposées ensemble le 31 octobre $2011^{1}$. Le texte équatorien fait état d'enjeux « nouveaux » et « urgents », et appelle à la proclamation d'une Déclaration Universelle des Droits de la Nature (section 2.17). Citant l'article 71 de la Constitution équatorienne, situé dans une partie dédiée aux droits fondamentaux, l'Equateur affirme que la reconnaissance de droits de la nature est « la reconnaissance d'un droit [de la nature] au plein respect de son existence, au maintien et à la régénération de ses cycles vitaux, de ses structures, fonctions et processus évolutionnaires » (notre traduction). La Constitution garantit par ailleurs l'ensemble des droits de l'homme, notamment le droit à un environnement sain, et la contribution à Rio +20 demande aussi de le faire.

Du côté de la Bolivie, la formulation est très proche : « les humains ne sont pas les seuls à

1 Texte téléchargeable ici : http://www.uncsd2012.org/rio20/comp_memberstates.html 
avoir un droit à une vie saine, les autres composants et espèces de ce système que nous appelons nature ont aussi ce droit. Dans un système interdépendant et inter-relié tel que la planète Terre, il n'est pas possible de ne reconnaître des droits qu'aux humains sans affecter l'ensemble. Tout comme les humains ont des droits, la Terre Mère a aussi le droit d'exister, le droit de maintenir ses cycles vitaux, le droit à la régénération, le droit d'être libre de toute altération structurelle, et le droit d'être reliée aux autres parties du système Terre. Pour rétablir l'équilibre avec la nature, il est nécessaire d'établir clairement les obligations que les humains ont envers la nature, et de reconnaître que la nature a des droits qui doivent être respectés, connus et défendus » (notre traduction).

\section{Le projet de Déclaration Universelle des Droits de la Terre-Mère rédigé à Cochabamba} stipule d'abord que « 6) tout comme les êtres humains jouissent des droits humains, tous les autres êtres de la Terre Mère ont également des droits spécifiques à leurs conditions et propres au rôle et à la fonction qu'ils exercent au sein des communautés dans lesquelles ils existent. 7) Les droits de chaque être sont limités par les droits des autres êtres, et tout conflit impliquant ces droits doit être résolu de façon à ce qu'on préserve l'intégrité, l'équilibre et la santé de la Terre Mère. ". Suivent ensuite deux articles, l'un relatif aux droits « inhérents » ou " intrinsèques » (" intrinsic rights ») de la Terre-Mère, l'autre aux obligations des êtres humains envers cette Terre-Mère. Le quatrième article indique que le « Le terme "être" [relatif à « tous les autres êtres de la Terre Mère »] comprend les écosystèmes, les communautés de nature, les espèces et toutes les autres entités naturelles qui existent comme partie de la Terre Mère. »

La position de la Bolivie indique se situer dans une continuité qui démarrerait avec la Charte Mondiale pour la Nature (1982). Cette charte ne mentionne ni « droits de la nature » ni même une " valeur intrinsèque », mais fait état des mêmes préoccupations : « la nature doit être respectée et ses processus essentiels ne doivent pas être dégradés » (point I.1.). Toute personne a le devoir d'agir en accord avec les provisions de la Charte (point III.24). La Déclaration de Rio ne fait pas non plus apparaître de droit de la nature ni de valeur intrinsèque ${ }^{2}$. Son premier principe énonce que « Les êtres humains sont au centre des préoccupations relatives au développement durable. Ils ont droit à une vie saine et productive en harmonie avec la nature ». La Déclaration stipule toutefois la nécessité de préserver « l'intégrité » de l'écosystème terrestre (Préambule et Principe 7). L'intégrité renvoie au maintien d'un certain ordre du tout dans la relation avec ses parties,

2 http://www.un.org/french/events/rio92/rio-fp.htm 
cet ordre ayant une valeur en lui-même. C'est bien ce à quoi renvoie la notion de « valeur intrinsèque ». II y a donc bien là encore l'idée d'une dignité de la nature à protéger. La Convention sur la Diversité Biologique, adoptée à Rio, fait explicitement état d'une « valeur intrinsèque » de la nature, par contre, dans son Préambule. C'est là semble-t-il la première occurrence de cette expression. Mais les textes de Rio sont plus « conservationnistes » que " préservationnistes » : ils traitent principalement de l'usage durable et équitable des ressources. En fait ils sont bien préservationnistes, mais de manière indirecte, puisqu'un « usage durable » ne peut se concevoir sans se soucier des conditions de durabilité de la ressource en elle-même. La Charte de la Terre (2000), enfin, évoque quant à elle un « respect » et le souci de protéger une " intégrité écologique » ; mais elle n'évoque ni valeur intrinsèque ni droits de la nature ${ }^{3}$. Néanmoins l'intégrité écologique est peu différente de la valeur intrinsèque, en substance, on l'a vu.

Les propositions boliviennes et équatoriennes introduisent donc bien quelque chose de neuf dans le débat international. Peut-être même le seul élément nouveau, dans le processus. La dégradation de la planète est malheureusement peu perceptible, elle n'exerce pas l'effet de sidération qui serait nécessaire pour que se crée ce que Lacan appelait un « point de capiton », ce par quoi le signifiant arrête son glissement indéfini sur la signification, et génère un point d'attache qui règle ensuite les actes, un moment à partir duquel jaillit un sens collectif - par exemple l'évidence selon laquelle le monde est maldéveloppé dans son ensemble, qu'on soit sous-développé ou surdéveloppé. Tant que le monde développé se perçoit comme apex et incarnation de l'humanité comme telle, c'est lui, le point de capiton qui attise les convoitises. L'objectif de coopération est donc peutêtre encore hors de portée. Ce qui pousse encore un peu plus vers cette interprétation est que le premier texte ("draft zero ») diffusé par l'ONU dans la perspective des discussions de juin est un catalogue de bonnes intentions, du type « les objectifs de Rio doivent être atteints, c'est important, alors que chacun fasse de son mieux » etc. Aucun diagnostic n'a été préalablement porté sur les raisons pour lesquelles rien n'a avancé depuis Rio 92, en matière d'environnement, ni pourquoi les réussites en matière de développement sont confinées pour l'essentiel à une petite partie de l'Amérique Latine et à l'Asie du Sud-Est, à l'exclusion de l'Afrique. Rien n'est dit sur la croissance du Quart-Monde dans les pays développés. De Rio il ne subsiste que quelques principes cruciaux, tel que celui des « responsabilités communes mais différenciées », qui n'ont aucune traduction concrète. Tout ce passe comme si ce discours vide était fait pour qu'il y ait quelqu'un à Rio en juin,

3 http://www.earthcharterinaction.org 
plutôt que rien. Et sur place on trouvera bien, se disent les rédacteurs du texte, le moyen de faire une déclaration de bonnes intentions. Mais rien n'aura été réglé sur le fond. Les ONG qui essaient d'influencer le texte final pourraient bien perdre leur temps. II n'est pas exclu que ce sommet soit surtout caractérisé par l'absentéisme des principaux responsables de l'état de la planète, qui n'ont pas besoin de ce sommet pour agir. Dans le domaine du commerce international I'OMC a pareillement été largement mise sur la touche au profit d'approches bilatérales.

Que faire ? Plusieurs documents ont été émis par diverses institutions et sont en discussion. L'un des derniers est le rapport du Programme des Nations-Unies sur l'Environnement (PNUE), qui tente de définir comment parvenir à une « économie verte », définie comme à basse teneur en carbone, « efficace » sur le plan de l'usage des ressources et « socialement inclusive $~_{4}$. II s'agit d'obtenir un bon indicateur de développement humain, en réduisant ou en s'autorisant à augmenter l'empreinte écologique, selon les pays.

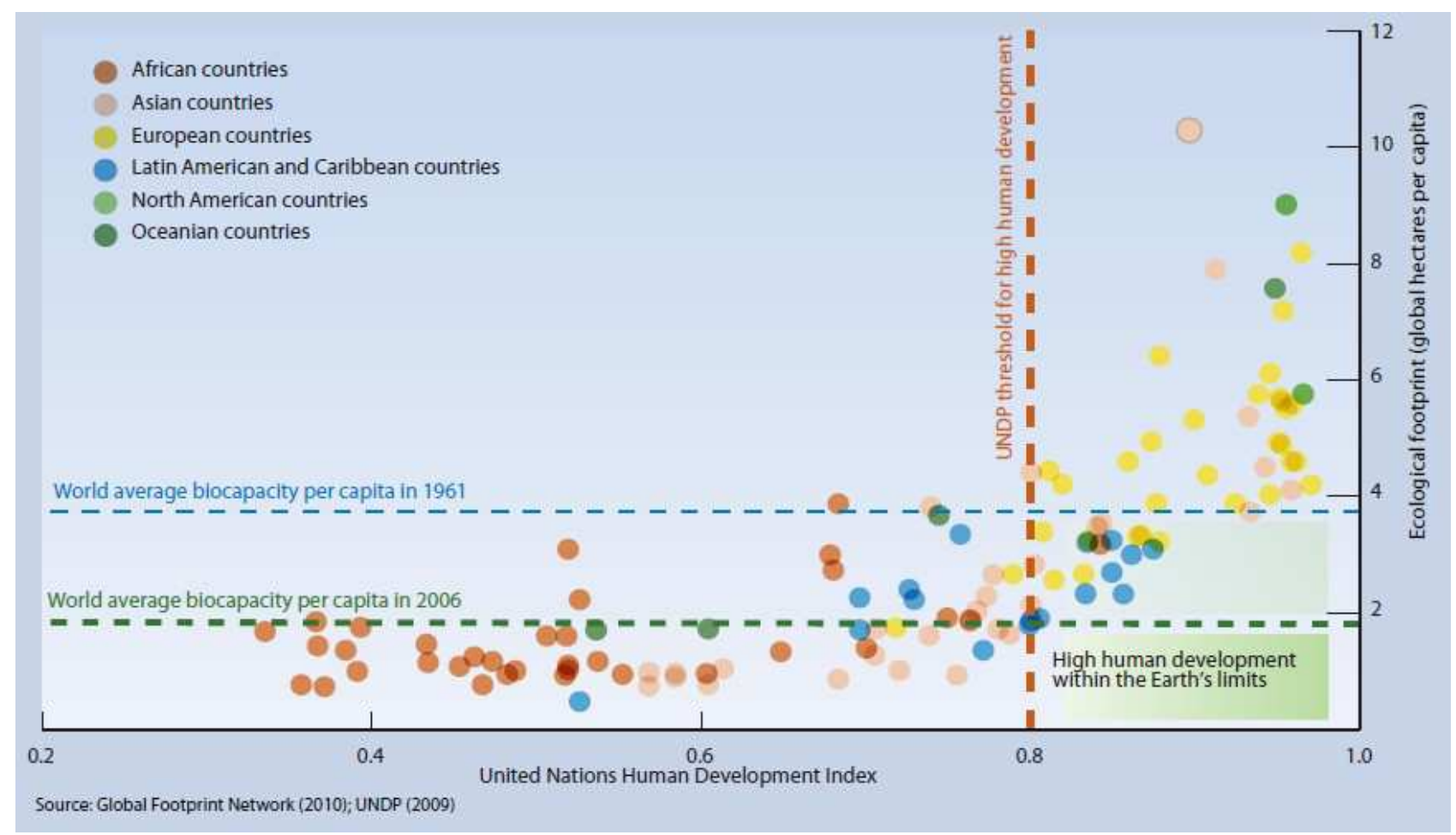

Source: PNUE, 2011.

Les « biens communs » planétaires seraient donc partagés et protégés. Ce programme semble aller dans le bon sens. Mais les moyens proposés pour y parvenir sont dérisoires, 
ils se ramènent pour l'essentiel à redresser les prix, par la régulation. Le texte vise donc plutôt à préserver la compétition économique, en rappelant aux gagnants qu'ils doivent faire preuve de magnanimité et qu'ils ont une obligation de protection envers les plus faibles. La télévision et Internet vont donc continuer d'exciter dans le monde entier la désirabilité du mode de vie "développé », à l'exclusion de tout autre, celui-là même qui nous enfonce lentement et collectivement dans le chaos, les $3 e$ classe en premier. La situation rappelle les deux duellistes du tableau de Goya, reproduit en couverture du Contrat Naturel de Michel Serres (1990), qui continuent de se battre alors qu'ils s'enfoncent lentement dans les sables mouvants. Jamais la coopération n'aura semblé aussi urgente, et simultanément hors de portée.

Le rapport sur la croissance verte publié par l'OCDE en 2011 entretient tout aussi savamment le flou dans les domaines clé de la négociation ${ }^{5}$. II évite soigneusement de définir ce qu'est la « vertitude » d'une économie, qualité laissée à l'appréciation des Etats - tout en appelant à la préservation des « biens publics mondiaux ». Dans ces conditions rien ne garantit que les politiques agrégées atteindront les objectifs fixés par le droit international, ni que la distribution des biens et des maux sera de nature à assurer les objectifs annoncés. Chacun pourra se dire « vert » pour la raison qui l'arrange le mieux la France en raison de son nucléaire, la Chine en raison de ses émissions par habitant, les Etats-Unis en raison de l'intensité carbone de son économie (kg CO2 / unité de PIB). Le problème est que ces différentes raisons ne convergent pas entre elles, et quand elles le font, ne convergent pas avec des objectifs écologiques ou distributifs. Prenons le cas de l'intensité en carbone, indicateur volontiers utilisé par l'OCDE. Un pays peut bien avoir une très mauvaise intensité carbone, s'il n'a que 10000 habitants, comme Tuvalu, ses émissions restent infimes. A l'inverse l'Inde et la Chine pourrait bien parvenir à la meilleure intensité carbone du monde que leurs émissions seraient quand même excessives, du fait de leurs trois milliards d'habitants réunis. Un tel critère est donc à l'évidence insuffisant. Mais le flou arrange tout le monde. Tant que la discussion continue, aucune décision ne se prend, et le business-as-usual est roi. Les dispositifs tels que REDD ou la « compensation carbone ", qui auraient pu être mis au service d'un développement " propre », comme indiqué, n'ont généralement pas d'effet démontré en faveur ni de l'environnement ni du développement ${ }^{6}$. Par contre les effets pervers sont bien là : spéculation, excuse pour les

5 OCDE, Vers une croissance verte, 2011. www.oecd.org/croissanceverte

6 Sur les réussites du Mécanisme de Développement Propre on lira notamment Sutter \& Parreno, Does the current Clean Development Mechanism (CDM) deliver its sustainable development claim? An analysis of officially registered CDM projects, Climatic Change, n॰84, 2007, pp. 75-90. Voir aussi Alexander Vasa \& Karsten Neuhoff, The role of CDM post-2012, DIW Berlin, Janvier 2011. 
pays en développement et pour les pays développés pour éviter de mettre en place des politiques nationales etc. La dérive générale vers la financiarisation menace les initiatives intéressantes de redistribution des richesses telles que CO2 Solidaire ou le Gold Standard.

Aucun des deux rapports cités ne se demande vraiment pourquoi la compétition mortifère continue, ni ce qu'il faudra pour qu'elle cesse. Les mouvements sociaux et ONG sont encore trop faibles pour l'endiguer. Dans ce contexte la Déclaration des Droits de la Nature est peut-être un espoir. L'attachement à la nature est peut-être notre seule chance, car il rend palpable ce que chacun doit aux autres, et permet ainsi d'établir un lien social cosmopolitique. II permet à une cosmologie planétaire d'exister. II crée un « point de capiton », comme disait Lacan, un élément qui réorganise entièrement notre vision du monde et de la vie. Respecter la planète c'est respecter l'Homme, parce que l'Homme a besoin de la nature. Telle est sans doute la portée de la proposition bolivienne de « Droits de la nature ». Dans l'espace international la dimension symbolique est importante. Adopter une déclaration sur les « droits de la nature », en complément des droits de l'Homme, serait un signal fort pour le monde entier.

\section{Une vieille revendication écolo}

Le fait que cette revendication soit issue d'un « Sommet des Peuples » largement plébiscité par les mouvements altermondialistes, et que ceux-ci se mettent brutalement à reprendre la revendication de droits de la nature, pose question. D'où vient cet engouement ? Pour des figures majeures des Forums Sociaux tels que Bernard Cassen, l'altermondialisme s'inscrit plutôt à la suite des mouvements ouvriers, socialistes, au sens large, c'est-à-dire y compris les socialismes du Tiers-monde. La revendication de « droits » pour la nature ne fait pas du tout partie du répertoire classique. Ce point provoque même l'indignation d'autres figures de l'altermondialisme français, tels Pierre Khalfa cité en introduction ou Jean-Marie Harribey ${ }^{7}$, à peu près pour les mêmes raisons : accorder des droits à la nature serait un non-sens car seul l'être humain peut aller plaider devant un tribunal, lui seul étant doté d'une subjectivité capable d'élaborer quelque chose comme des droits ; ce serait contraire à l'humanisme des droits de l'homme et réactiver un holisme prémoderne, réinscrivant l'homme dans un ordre naturel etc.

7 Jean-Marie Harribey, La nature sujet de droit : une fiction, un mythe fondateur pour changer la réalité ? 
Comme le montre plus de quatre décennies de débats en matière d'écologie politique, le « droit de la nature » est l'élément clivant de ce courant vis-à-vis des autres courants politique. La proposition de C.D. Stone, l'avocat du Sierra Club, une organisation écologiste étasunienne, d'attribuer des droits aux arbres, pour pouvoir contrer le projet de Disney $^{8}$, en 1972, est souvent considérée comme inaugurale. La même année, ClaudeMarie Vadrot, relatant la tenue du Sommet sur l'environnement, à Stockholm, avait évoqué une « déclaration des droits de la nature » pour caractériser le message essentiel des écologistes de l'époque ${ }^{9}$. Et les clivages bien connus étaient déjà là, à cette époque. Les écologistes s'étaient opposés aux grandes entreprises (dont le Club de Rome), aux gouvernements des Etats riches, mais aussi au Parti Communiste, grand promoteur du " progrès », et des syndicats. Les écologistes s'étaient retrouvés aux côtés des " pays en voie de développement ». Des pays en développement dont certains semblent vouloir reprendre la main, désormais, non sans contradictions d'ailleurs - on lira dans Mouvements les articles publiés sur le « néo-extractivisme », en gardant en mémoire que les pays les plus extractivistes sont d'abord, et de loin, les pays développés, et qu'il est peut-être déplacé de notre part de les stigmatiser ou pointer leurs inévitables contradictions. Bref. Rio +20 représente d'abord, pour les riches, le risque de se voir contester ce privilège qui leur permet d'assurer leur train de vie. On se souvient de la phrase de Bush père, à Rio : « notre mode de vie n'est pas négociable ». Sous-entendu : et nous irons faire la guerre s'il le faut pour continuer à extraire les ressources, telles le pétrole irakien, qui nous permettent de continuer à dominer.

La revendication de « droits de la nature », loin de surgir du néant, s'inscrit donc en réalité dans une " troisième vague » écologiste, après celle des années 70 et celle des années 90. Le fait que la revendication vienne de pays sous-développés est un démenti flagrant à ceux qui soutiennent que ces pays souhaitent avant tout imiter le Nord et n'ont pas d'autre visée que le développement économique - bref, que l'homo economicus est universel, mais qu'il aurait été pendant des millénaires enfoui sous la Tradition. Que cette revendication soit issue de pays à forte coloration « indigéniste » (Morales, Correa) est peu surprenant, il n'a pas échappé aux observateurs attentifs que les écologistes ont de leur côté toujours été de fervents soutiens des peuples autochtones. Que cette idée de « droits de la nature » ait pu être en partie soufflée à la Bolivie et à l'Equateur par une ONG étasunienne, le Community Environmental Legal Defense Fund, ne retire rien à ce constat. En témoigne la célèbre déclaration du chef Seattle, qu'on retrouve souvent citée 
dans la littérature écologiste : « nous savons au moins ceci : la terre n'appartient pas à l'homme ; I'homme appartient à la terre. Cela, nous le savons. Toutes choses se tiennent comme le sang qui unit une même famille. Toutes choses se tiennent. Tout ce qui arrive à la terre, arrive aux fils de la terre. Ce n'est pas l'homme qui a tissé la trame de la vie : il en est seulement un fil. Tout ce qu'il fait à la trame, il le fait à lui-même. ». Que le chef Seattle ait réellement tenu ces propos est de peu d'importance, en définitive, ce qui compte est que ces mots parlent à ceux qui souhaitent les entendre, et qu'une alternative soit possible.

Cette troisième vague sera-t-elle la bonne ? La première, qui avait vu l'émergence du mouvement, et la candidature de René Dumont, déjà partisan d'une écologie « socialiste », s'était délitée sur « les années fric », les années 80 , au cours desquelles ce qui s'était produit en France n'était pas pas le socialisme mais la mondialisation capitaliste, achevée par Jospin, sur le plan théorique, qui affirma de son programme qu'il n'était pas socialiste. La seconde vague est liée au sommet de Rio, en 1992. Elle a suscité une percée aux élections, notamment aux européennes ${ }^{10}$ - mais aussi la mise en place de contre-feux tels que le livre de Luc Ferry, Le Nouvel Ordre Ecologique (1992), sur l'argumentation duquel nous reviendrons plus loin. La gauche plurielle et l'émiettement écologiste, en partie provoqué par Mitterrand, affaiblissent le mouvement par la suite, avant ce retour auquel nous assistons aujourd'hui. La situation a en effet beaucoup changé. La question environnementale s'est en effet inscrite dans le quotidien, partout dans le monde. En France le Grenelle, s'il n'a pas débouché sur des changements concrets, a joué un rôle de déclencheur pédagogique. Le niveau de connaissance de la population a fortement augmenté. Fukushima et les gaz de schiste ont aussi contribué à médiatiser les questions énergétiques. Dans le même temps le néolibéralisme a perdu de sa puissance, se montrant incapable de réguler les crises qu'il engendre. Les écologistes, s'étant fortement institutionnalisés, ont tardé à prendre la mesure de ces changements et un certain radicalisme est revenu à l'ordre du jour, se manifestant en France par les courants de la décroissance.

« L'écologisation » des sociétés est en marche. Partout, dans le monde, se diffuse la connaissance écologique, même si cela ne conduit pas directement à l'action. Un grand quotidien indien, l'Hindustan Times, se félicitait, en 2008, d'être classé par l'indicateur Greendex, conçu par le National Geographic, pays le plus vert. La couverture médiatique 
du sommet sur le climat de Copenhague, en 2009, a été bien meilleure en Inde qu'en France, comme l'auteur de ces lignes peut en témoigner. Le tournant écologiste de l'altermondialisme est un autre signe de cette mutation en cours. L'écologie était jusque-là en marge d'un mouvement dont Bernard Cassen estimait que « tout a commencé à Porto Alegre $»^{11}$, méconnaissant ainsi les contre-sommets écologistes qui ont précédé de 30 ans le Forum Social Mondial. Des observateurs tels Jean Jacob ont entériné cette vision, distinguant soigneusement « l'altermondialisme », qu'il estime de gauche, progressiste, de ce qu'il appelle « l'antimondialisme », qu'il juge plus conservateur, où il s'avère que la plupart des personnes mentionnées (Vandana Shiva, Edward Goldsmith etc.) sont des altermondialistes écologistes ${ }^{12}$. Stigmatisé en raison de sa critique de la modernité, l'écologisme est resté en marge. Mais avec la manifestation géante organisée autour du sommet de Copenhague sur le changement climatique (2009), écologisme et « altermondialisme » se rapprochent jusqu'à fusionner - ce dont les écologistes, de leur côté, n'ont jamais douté. Le succès du Sommet de Cochabamba sur la Terre-Mère, organisé par la Bolivie à la suite de l'échec de Copenhague, ne saurait mieux démontrer ce virage de «l'altermondialisme » à la Cassen.

Ce qui est surprenant, ou récurrent, c'est selon, est que les mouvements de gauche classique s'opposent à cette perspective, cela pour des raisons qui sont très proches de la droite. Parler de « droits de la nature » est en effet déjà ce qui avait attiré les foudres de Luc Ferry et de Dominique Bourg ${ }^{13}$, successivement. Pour eux il y aurait tout au plus lieu de parler de « devoirs » de l'homme vis-à-vis d'une nature sur laquelle l'homme continuerait d'avoir tous les droits, en en faisant bon usage - solution aussi choisie par Jean-Marie Harribey (art. cité). Comment expliquer cette curieuse convergence ?

III. Accorder des droits à la nature : absurde ? Contraire à l'humanisme ?

Marie-Angèle Hermitte, qui travaille depuis vingt ans sur les questions de droit de la nature, en convient : le droit moderne, d'un point de vue technique, ne pose aucun problème à la constitution de la nature en sujet de droit ${ }^{14}$. Les juges de la cour suprême des Etats-Unis avaient d'ailleurs partiellement soutenu la proposition inaugurale de C.D.

11 Bernard Cassen, Tout a commencé à Porto Alegre, Mille et Une Nuits, 2003.

12 Jean Jacob, L'antimondialisation, Berg, 2006. Voir http://www.mouvements.info/L-antimondialisation-Aspects.html

13 Dominique Bourg, Les scénarios de l'écologie, Paris, Hachette, 1996.

14 Marie-Angèle Hermitte, La nature, sujet de droit? Annales. Histoire, Sciences Sociales, 2011/1 (66e année), pp173-212 
Stone $^{15}$. Le problème ne vient pas de là. Elle montre aussi qu'il est généralement plus facile de protéger la nature pour ce qu'elle est, en raison de sa dignité intrinsèque, que de chercher à lier un état de la nature à un intérêt humain, comme le fait habituellement le droit moderne. La revendication de « droits de la nature » se justifie donc très bien sur un plan pragmatique. C'est une solution efficace, chacun peut ainsi constater les atteintes à l'intégrité de la nature et s'ériger en représentant de la nature pour la défendre devant un tribunal, c'est du reste exactement ce que prévoit la Constitution équatorienne. Toute autre solution se révèle au contraire malaisée et difficilement praticable, comme le démontre le peu de progrès fait dans ce domaine depuis plus de quatre décennies. Les associations se sont donc à juste titre félicitées d'obtenir la reconnaissance, lors du procès de l'Erika, de l'existence d'un « préjudice écologique pur ». Rien n'indique, à première vue, pourquoi une telle perspective, qui ferait de chaque citoyen un défenseur de la nature, pose de tels problèmes.

Les critiques ne s'intéressent pas beaucoup à ces éléments pragmatiques. De l'avis de tous les spécialistes, Luc Ferry n'a qu'une connaissance très superficielle des questions écologiques concrètes, son livre recèle de nombreuses inexactitudes. Sa conclusion, selon laquelle « réconciliée avec l'Etat, qui lui donne des ministres, avec la démocratie, qui offre la possibilité de changements sans violence, l'écologie s'intègre enfin au marché, qui s'adapte tout naturellement aux exigences des consommateurs » (p. 215), se désintéresse de sa propre faisabilité. Et si l'intégration de l'écologie était au contraire profondément antagonique avec la démocratie libérale et le marché ? C'est un soupçon qui pèse depuis longtemps sur les écologistes, perçus à droite comme des « pastèques » (vert à l'extérieur, rouge à l'intérieur) et à gauche soupçonnés d'être porteurs d'une idéologie secrètement réactionnaire. La suggestion alternative faite par Jean-Marie Harribey, d'un choix politique conscient, « rationnel », n'est pas plus explicite. Quel serait le critère de la décision rationnelle ? Et en quoi cette rationalité serait différente de celle qui est établie, dans la relation à la nature ? Se refuser à déterminer cette rationalité, en en laissant la responsabilité à la démocratie et au débat, ne résout pas le problème, ça l'escamote. Car rien ne prouve que les débats démocratiques vont s'organiser autour de quelque chose qui ne serait absolument pas théorisable. Refuser de théoriser la principale revendication écologiste a quelque chose de dérangeant, c'est comme si l'on récusait, dans le marxisme classique, toute prétention de la valeur-travail à une quelconque validité, au profit « du débat ». Car il y a bien quelque chose à penser ici. Il est remarquable, en effet que la 
proposition écosocialiste la plus en vue, celle de J.B. Foster, se propose pour programme de dépasser l'écocentrisme, qui caractérise la « green theory », selon lui - et cela sans y parvenir ${ }^{16}$. L'écocentrisme, c'est à la fois la revendication clé de l'écologisme et le point que les socialistes voudraient bien voir dépasser. Qu'est-ce qui gêne à ce point ?

Un premier élément de réponse vient de la référence à Kant, qui revient dès lors qu'il est question de « dignité ». Kant n'accorde de dignité qu'à l'être humain. Marie-Angèle Hermitte constate que le droit moderne est cartésien, en plus d'être kantien : pour lui n'existent que des res cogitans qui peuvent librement disposer de la res extensa pourvu que les droits des autres res cogitans soient respectés. Ce schéma fonctionne bien pour autant que le déplacement et la redisposition des parcelles de res extensa, pour faire des villes et des autoroutes, par exemple, engendrent des effets généralement considérés comme positifs, limitant ce que l'économie appelle les « externalités négatives ». Les diverses théories du développement se sont toutes appuyées sur cette ontologie, fondement de la croissance ${ }^{17}$. L'instrumentalité absolue de la nature est l'exacte contrepartie d'une organisation sociale « individualiste », fondée sur le contrat social, qui n'engage en rien envers la nature, par définition. Dans ce paradigme ce qui lie les humains est l'échange (verbal, monétaire, économique), issu d'un travail spécifiquement conçu comme " gain de temps ${ }^{18}$, et sa règle (ou « organisation »). Or la caractéristique de la crise écologique est que ce « gain de temps » comme critère de la rationalité industrieuse est plus en plus destructif. Ce n'est pas seulement un problème de réglementation et « d'excès » mais une mise en cause de cette rationalité ainsi que de l'ontologie sociale qui l'accompagne, en tant qu'elle est incapable prendre en compte « la nature » entendue comme ensemble d'écosystèmes et de populations non-humaines, une nature dont nous dépendons pourtant pour notre accomplissement et notre « développement ». C'est le caractère absolument instrumentalisable de la nature qui est en cause. D'où la revendication de « droits de la nature », au sens d'une obligation de respect des non-humains (biocentrisme) ou de certaines formes des écosystèmes (écocentrisme). La dignité de ces éléments est reconnue, là où le droit anthropocentrique ne reconnaît de dignité qu'aux res cogitans.

Ce glissement hors du kantisme et du cartésianisme soulève toutes sortes de questions,

16 Fabrice Flipo, L'écologie politique est-elle réactionnaire-l'enjeu des choix technologiques chez John Bellamy Foster, Sens Public, 16 juin 2010. http://www.sens-public.org/spip.php?article754

17 Yves Berthelot, Unity and diversity in development ideas - perspectives from the UN Regional Commissions, Indiana University Press, 2004.

18 Jacques Bidet, Théorie générale, Paris, PUF, 1999. 
qui relèvent souvent d'un procès en anthropomorphisme. Habitués à raisonner dans le cadre cartésien, les critiques imaginent ce que ce cadre produirait si, à la place de l'Homme, on mettait les animaux ou les écosystèmes : comment la nature va-t-elle plaider dans un tribunal ? Est-ce que ça ne revient pas à attribuer une âme à la nature ? Etc. Pourtant ces objections sont largement dénuées de fondements : aucun partisan des « droits de la nature » (ni du droit des animaux !) n'a jamais soutenu que ces droits devaient être rigoureusement identiques aux Droits de l'Homme. Tous ont expliqué que de tels droits devaient être adaptés à l'entité visée, comme le sont d'ailleurs les droits de l'Homme eux-mêmes. Les animaux n'auraient donc pas les mêmes droits que l'homme. L'exercice de ces droits ne peut se faire qu'au travers d'une représentation. Que l'on se voie accorder des droits n'implique pas qu'on soit capable de les porter en propre, comme le montre le cas des enfants ou des malades qui ne peuvent exercer les leurs - ce parallèle a souvent été fait. L'important est qu'un droit puisse être opposé à un autre droit, de manière à pouvoir instituer une borne. Et de ce point de vue les droits de la nature sont bien une limite aux droits de l'homme, du moins quand ceux-ci sont mis en œuvre de la manière critiquée par Marx dans La Question Juive : comme droit de l'homme égoïste, qui ne se soucie pas de la communauté. Sauf que dans ce cas c'est de la communauté biotique dont il s'agit. L'humanisme moderne se confond avec le droit absolu de l'Homme sur cette communauté biotique, c'est en ce sens précis que la revendication de « droits de la nature » peut s'assumer comme « anti-humaniste ». Ce geste prolonge celui d'Althusser, qui avait lui aussi préconisé un anti-humanisme théorique, au service d'un humanisme pratique. L'humanisme pratique des partisans des « droits de la nature » est que le monde n'est pas humain s'il n'accorde aucune dignité aux êtres de nature. Est humaniste celui qui entend défendre un rapport humain au monde, et non un rapport barbare ou d'exploitation. On lira pour s'en convaincre l'interview réalisée dans ce numéro avec un représentant du peuple Sarayaku.

Si l'on s'accorde avec cela alors on peut encore objecter du vocabulaire employé : ne peut-on parler de « devoirs », et non de « droits », pour désigner ces obligations, afin d'éviter toute confusion anthropomorphiste ? II est intéressant de se rappeler, dans ce contexte, que ceux qui défendaient les « devoirs de l'homme », face aux partisans des « droits de l'homme », pendant la Révolution, étaient les conservateurs. Leur argument était que l'homme ne devait pas oublier sa dépendance à « la société », c'est-à-dire en fait son obéissance aux autorités. Le devoir de l'Homme rappelait le devoir d'obéissance et le risque que les Droits de l'Homme présentaient pour les autorités établies, qui étaient 
tenues de se soumettre à ces droits, qui priment sur « la société » au sens d'une totalité organique figée, d'un ordre établi. Or tel semble aussi être l'objet du « droit de la nature » : c'est avant tout contre l'ordre établi qu'ils sont mis en avant. L'expression « droits de la nature » semble donc adaptée, de ce point de vue. Parler de « devoirs » ne résoudrait rien, de toute manière, sur le fond, si l'enjeu est de reconnaître une dignité à la nature : changer de signifiant (de « droit » pour « devoir ») ne résoudrait pas ce problème de signifié. Or sans dignité de la nature, pas de protection de la nature. Tout semble donc confirmer la rationalité de la revendication de « droits » pour la nature.

Un dernier point reste toutefois à soulever. Eviter l'anthropomorphisme et les divers problèmes soulevés ici ne nous dit pas qui parlera au nom de la nature et quand. II est remarquable à ce sujet que le texte bolivien mette en avant le fait que l'instauration de « droits de la nature » devrait permettre à chacun, notamment les mieux placés pour constater la violation des droits, d'ester en justice contre des actions qui contreviendraient à ces droits. Les expertises en la matière seront forcément multiples mais elles trouveront enfin un lieu pour se confronter. Les savants n'auront plus le privilège de dire les droits de la nature. La possibilité épistémologique établie par Michel Serres, de passer du droit au fait et du fait au droit, se trouve confirmée mais aussi et surtout démocratisée potentiellement, car évidemment tout dépend de la mise en oeuvre, qui fasse que ces droits ne soient pas seulement formels. La solution est beaucoup plus séduisante que celle de Bruno Latour, par exemple, qui appelait à multiplier « les natures », la nature en tant que telle étant une entité qui « éviscère le politique ${ }^{19}$, « une bizarrerie stratégique [chez les écologistes] qui rend impraticable le combat politique ». Ce que Latour n'a pas vu est qu'en mettant tout en politique, c'est la possibilité même d'un débat qu'il a dissous. Aucun débat ne s'organise à partir de rien, tout débat commence à partir d'un cadre qui se pose comme un donné. Ce cadre est celui à partir duquel un débat est possible. II comprend notamment le droit et les procédures de débat, mais pas seulement : tout ce qui est intelligible en fait partie. « La nature » est une partie de ce cadre, on ne peut la fractionner sans aussitôt fractionner le cadre, et donc le collectif qui débat.

\section{La modernité en question}

Une seconde question se pose, toutefois, et Jean-Marie Harribey a raison de la poser, de

19 Bruno Latour, Politiques de la nature, La Découverte, 2004, p. 35. 
savoir si sortir du kantisme, et donc peut-être de la modernité, nous ramène aux sociétés non-modernes, à l'âge de pierre, à l'époque où la nature semblait « enchantée » - bref, au Chef Seattle. Cette question lancinante habite la problématique écologiste depuis le départ, et n'a pas, en pratique, de réponse tranchée. Il est extrêmement dérangeant, pour les pensées occidentales qui se réclament du progrès et de l'universalisme, de constater que les peuples désignés par la sociologie occidentale comme " primitifs " soient détenteurs d'une vérité écologique qui assure leur pérennité, pour la plupart d'entre eux, alors que nous Modernes sommes menacés de disparition. La question ne peut donc pas être évitée. Demander de quoi le « bien-vivre » est fait est donc une question clé.

Socialisme et communisme peuvent être diversement définis mais ils ont en commun de ne pas faire l'apologie du monde précapitaliste, appréhendé comme un lieu d'aliénation. La Théorie Générale de Jacques Bidet, par exemple, qui fait suite à sa Théorie de la modernité (1990), fait de la déclaration de liberté-égalité-rationalité universelle une caractéristique de la modernité, laquelle émerge avec le capitalisme. La modernité est « une logique sociale d'ensemble, qui se déploie sur près d'un millénaire à partir de l'Europe occidentale ». La liberté renvoie à deux dimensions : la « coopération productive ", marchande (liberté des Modernes) et organisationnelle (liberté des Anciens), toutes deux fondées sur un refus de la naturalité des règles (« thèse de la règle » §112). L'organisation émerge en raison des « coûts de transaction », fruit de la rationalité, mais aussi en raison de l'établissement de règles, pour articuler les différentes libertés et en particulier les égaliser au sens de les justifier par un accord discursif universel. Ainsi naissent les nations avec leurs caractéristiques propres (culturelles linguistiques historiques etc.). « Non seulement le « capitalisme » instaure, pour la première fois, entre nations un espace public. Mais c'est par cela même aussi qu'il y a nations. L'empire n'a pas à se justifier ainsi devant les barbares [...] La paix moderne se distingue de la pax romana. Elle n'est pas moins hégémonique ni moins massacreuse. Bien au contraire. Mais elle se réfère à, et dit disposer sur, des principes acceptables par tous » ( $\$ 613$ p247). D'où ce fait que l'horizon du capitalisme est le monde entier, d'où l'émergence d'une étaticité mondiale (\$622), d'une « ultimodernité ». Le libéralisme et le capitalisme se caractérisent par une dénégation de la dimension organisationnelle et un refus de tirer les conséquences de cet accord discursif dont ils se réclament pourtant. Le socialisme au contraire prend au sérieux cette exigence d'universel (\$922). Pour Bidet, l'écologie pose la question d'une détermination démocratique d'un usage cohérent d'un environnement fini (\$632 p299), « de la considération écologique ne suit pas l'exigence de produire moins, 
mais seulement une contrainte rationnelle à emprunter des détours (intellectuels) de production excluant ces externalités négatives, soit une invitation à travailler plus longtemps pour le même résultat » (\$931 p466).

Cette position pose bien des problèmes. Le premier est que Bidet ne tire pas véritablement les conséquences de sa propre position. A quel genre de société conduirait l'application de la « considération écologique » ? Comment peut-il affirmer qu'elle ne conduit pas à « produire moins » ? Pour universaliser le mode de vie européen il faudrait diviser notre consommation par trois ou quatre, comme le suggère un rapport des Amis de la Terre déjà ancien ${ }^{20}$. Sortir du nucléaire, diviser par cinq nos émissions de gaz à effet de serre ne peut se faire sans « sobriété », nous dit le dernier scénario négawatt, c'est-à-dire qu'il ne peut se faire sans produire moins. Jean-Marc Jancovici a brossé le portrait d'un monde énergétiquement vertueux, mis à part le biais pronucléaire l'exercice est intéressant et montre qu'on devrait se situer sur une trajectoire tout-à-fait différente de celle que nous suivons actuellement ${ }^{21}$. Il se produirait une contraction des échanges internationaux qui mettrait en péril la perspective offerte par Bidet de l'émergence d'une étaticité mondiale, via le capitalisme. Le récit d'une telle évolution semble donc échapper à sa propre exigence d'universalisabilité et faire le jeu d'une certaine élite internationale, qui profite largement du capitalisme mondialisé. Ce récit se désintéresse aussi de la « clause de Locke ", qui exige que toute consommation de ressources naturelles en laisse autant et d'aussi bonne qualité à tous les ayant-droit : « nul ne [peut] s'estimer lésé de voir une autre personne boire, même à pleine rasade, s'il lui laissait toute rivière de la même eau pour étancher sa soif. Ce qui vaut pour l'eau vaut identiquement pour la terre, s'il y a suffisamment des deux ${ }^{22}$. Cette clause est pourtant l'enjeu de la divergence entre libertarisme de droite (Robert Nozick) et libertarisme de gauche (Peter Vallentyne). Pour les premiers l'appropriation des ressources naturelles est privée et chacun doit se débrouiller pour trouver des ressources à valoriser ; tandis que pour les seconds tout le monde a droit à une part minimale, ce qui les conduit à soutenir l'idée d'une allocation universelle, étant entendu que dans nos sociétés industrielles ne distribuer que de la terre n'aurait pas toujours une grande pertinence. A l'échelle mondiale l'allocation universelle exigerait une réduction drastique de la part appropriée par les plus riches, cette quête d'égalité dans « l'espace écologique » de notre existence est d'ailleurs au centre des désaccords dans les négociations sur le changement climatique. La justice climatique,

20 Wuppertal Institute, Europe soutenable, 1995. www.amisdelaterre.org/Rapport-Vers-une-Europe-soutenable.html $21 \mathrm{http}: / /$ www.manicore.com/documentation/sobriete.html

22 John Locke, Deuxième Traité du gouvernement civil, Paris, Vrin, 1985, Ed. Orig. 1690, Chapitre V. 
c'est attribuer à l'atmosphère le statut d'une res communis, une chose commune à laquelle tout le monde a droit, mais un droit qui ne dépasse pas celui des autres ${ }^{23}$. Ce qui vaut pour le climat vaut pour d'autres biens communs.

N'admettre la nature que comme « une contrainte rationnelle » est un second problème. De quelle « contrainte » s'agit-il ? Ce terme semble désigner une contrainte physique. Et en effet tel est bien le cas de l'épuisement des ressources souterraines et minières. Le « pic de pétrole » est inéluctable. L'entropie est un fait physique que l'ouverture de la Terre au flux solaire ne réfute pas, elle ne fait que l'atténuer. II reste à en tirer les conséquences, sur le plan de l'universel, à nouveau. Mais ce n'est pas tout. Si des droits de la nature sont nécessaires, c'est parce que la nature est fragile : nous pouvons changer le climat, vider les océans, exterminer les espèces etc. Ce n'est pas l'impénétrabilité de la nature qui nous en empêche, ici. La « contrainte » n'est donc pas d'ordre matérielle. Elle est d'ordre morale et symbolique. Pour qu'elle existe, quelque chose doit nous obliger, au sens moral. Et c'est effectivement ce qui se produit si la nature se voit reconnaître une dignité propre. Envisager la nature comme un obstacle c'est se préparer à sauter l'obstacle, au contraire. C'est inscrire le rapport à la nature dans l'ordre de l'instrumentalité. Au contraire la thèse des « droits de la nature » considère que la nature est aussi fin en soi - autrement dit, une richesse. Elle remet en cause la cosmologie moderne et porte un autre regard sur le monde. Les craintes des Modernes, à nouveau, sont significatives : ne va-t-on pas régresser vers l'animisme ? L'homme va-t-il être réinscrit dans un « ordre naturel »? L'écologicité du monde se trouve niée : entre la res cogitans et la res extensa, il n'y a rien. Toute tentative pour voir autre chose est rabattue sur l'une ou sur l'autre - voir la res extensa comme une res cogitans est de l'animisme, voir la res cogitans comme une res extensa est une réification dans un « ordre naturel ». La dichotomie moderne est redoutable et réfute la tentative écologiste de faire société avec le non-humain, que ce soit au motif de sa sensibilité, de son intelligence relative, de son droit à la vie (animaux, biocentrisme) ou au motif que cet « ordre naturel » est déjà humain et n'a pas besoin d'être transformé pour l'être davantage, au contraire (stabilité climatique etc. écocentrisme).

Plus généralement on est frappé de l'absence, chez Jacques Bidet, d'une théorie critique de la valeur d'usage sous le capitalisme. La domination ne se manifeste que sous la forme de l'extraction de la plus-value (domination des propriétaires des moyens de production) et 
du « cadrisme » (domination des compétents p198). Et si l'indifférence du capitalisme par rapport aux valeurs d'usage est stigmatisée (p182) Bidet lui attribue pourtant en creux un effet positif, une « ruse de la raison » : celui de développer des « centricités " toujours plus vastes ( $\$ 622$ p278), réglant des quantités toujours plus larges de populations sous une même législation. La perspective est celle d'un Etat mondial. Bidet affirme que I'humanité ne connait son essence générique, sous la forme d'une volonté générale planétaire, qu'à la suite d'un long développement des forces productives (§622 p275). La proposition de « droits de la nature » considère que cette essence générique requiert de tout autres "forces productives ", à commencer par celles de la nature, et que la ruse de la raison que Bidet voit à l'oeuvre est peut-être un trompe-l'oeil généré par le capitalisme lui-même. Certaines destructions écologiques réalisées par le capitalisme au cours de sa reproduction élargie n'ont pas de versant positif. Aucune « ruse de la raison » n'est à l'oeuvre ici, il s'agit de pertes sèches, sans contre-partie. Le développement des forces productives qui est nécessaire pour parvenir à un Etat mondial pourrait bien exiger l'élimination d'une grande partie de la population. C'est une petite minorité, dans le temps et dans l'espace, qui aura bénéficié de cette expansion. Au moins cet universalisme devrait-il s'interroger sur le particularisme qu'il légitime. Qu'un dépassement dialectique ait lieu un jour ne va pas de soi. La thèse du destin mondial du capitalisme se confond avec l'auto-justification capitaliste. Soutenir qu'une centricité mondiale est requise pour que l'essence générique de l'humain, qui est d'être en harmonie avec la nature, se manifeste, est peut-être faux. Au contraire reconnaître la dignité de la nature, c'est nous reconnaître comme humains. Donc relocaliser, c'est universaliser. La cosmopolitique écologiste, fondée sur la res communis, se fait de proche en proche, à la manière de la contractualité interindividuelle, sans avoir besoin d'attendre l'unification d'un centre. Elle est donc pluraliste, du point de vue qui a été développé par Jacques Bidet, d'où son insistance sur le respect de la (bio)diversité culturelle.

Cette perspective diverge profondément de celle de Jacques Bidet, bien entendu - mais aussi de la perspective libérale, puisque pour Bidet le socialisme n'est rien d'autre que la réalisation de ce que le libéralisme ne fait que promettre. L'introduction d'une tierce entité, entre les res cogitans et les res extensa, est postmoderne. Emerge notamment un concept postmoderne de travail, que Jacques Bidet définit adéquatement comme celui qui aurait « cessé de trouver dans le marché et le plan les formes supérieures de son efficacité » (p70). Introduire la nature, en effet, échappe au plan comme au marché, puisque ceux-ci restent ancrés dans l'ontologie sociale moderne. Le postmoderne, affichant sa sortie de la 
modernité, pose naturellement la question de sa différence d'avec le prémoderne. L'écologie, nouveau progrès ou retour en arrière ? Les pratiques « écologiques » se distinguent par un rapport distancié à la technologie, aux « forces productives » restées trop peu critiquées par le marxisme. Un rapport qui se cherche (que faut-il penser d'Internet, par exemple ?...) mais qui a la reconnaissance de la nature comme fil conducteur. D'où l'importance stratégique de ce contact avec la nature - nature en ville, trame verte etc. Cette reconnaissance, en creux, assigne une place à l'être humain. Mais la rationalité économique, qu'elle soit égalitaire ou exploitatrice, ne le faisait pas moins sauf à quitter les rives d'un sain matérialisme. Dire que la nature est res extensa, ce n'est pas nier ni son existence ni sa résistance, ce n'est donc pas dire que l'homme est " acosmique », hors-nature au sens matériel du terme, c'est seulement la réduire à l'une de ses propriétés, et laisser entendre (sans le démontrer) que la modernité serait la manière universelle (et donc la seule) de s'en arranger. En disant cela la modernité peut être comprise comme " ne pensant plus », comme commettant cet " oubli de l'Etre » qui la conduit à n'entrevoir le monde que sous le mode de l'étant, de l'exactitude - de là l'intérêt qui se manifeste envers certains textes de Heidegger, et nous ne chercherons pas à savoir ici si une telle interprétation est tenable. Notons simplement, en forme de conclusion, qu'une telle ouverture cosmique peut être comprise comme proprement « cosmopolitique », et ce n'est pas un hasard si cette interprétation est aussi au fondement des études postcoloniales - Dipesh Chakrabarty s'appuie ainsi sur Marx et Heidegger pour chercher à " provincialiser l'Europe ${ }^{24}$ - ni si elle suscite un intérêt du côté des mouvements « indigénistes ». Nul relativisme dans cette approche : ce n'est que la raison hégélienne qui se remet en marche, déconstruit ses propres présupposés, et cherche à fondre un nouvel universalisme.

\section{Conclusion}

Cet article n'épuise pas la thématique des droits de la nature. Il y a toutefois plusieurs accusations dont on voit maintenant qu'elles ne tiennent plus, au terme de cet exposé. Parler de " droits de la nature » ne revient pas à inscrire l'Homme dans une essence, une nature fixe qui lui ôterait sa liberté. Dire cela, c'est comme dire que l'Homme serait figé, dès lors qu'il respecte ses semblables. En effet l'ordre écologique reste sous-déterminé, tout comme l'ordre social. Est « écologique », en réalité, ce qui est issu d'une praxis 
militante, et non ce qui est le produit d'une science. Si l'être humain est tenu de ne plus considérer la nature comme un simple moyen, il n'est pas tenu de sacraliser la nature entière non plus, et donc de s'en exclure. Tout, dans la nature, n'a pas à être sorti de la relation instrumentale. Les droits de la nature doivent s'articuler avec les droits de I'homme, qu'ils ne remplacent pas. Ils peuvent d'ailleurs être instrumentalisés, comme ces derniers, et servir de masque à l'égoïsme. Les droits de la nature peuvent être opposés aux pauvres et pas aux riches, dans une démarche malthusienne, de même que les droits de l'Homme peuvent être le masque de la propriété. Néanmoins, comme les droits de l'homme, ils peuvent être opposés aux pollueurs et exploiteurs pour les contraindre à réduire leur activité. Ils ont donc une valeur « déclarative » et discursive qui n'est pas à négliger. Enfin leur post ou antimodernité, bien que constituant un apparent handicap dans les zones les plus modernisées, permet aussi d'ouvrir ce dialogue global qui manque tant, dans des échanges internationaux qui se ramènent trop souvent à la diffusion d'un seul modèle : occidental, sous différentes variantes. 